\title{
Die EU-Kinderarzneimittelverordnung von 2007: eine Erfolgsgeschichte!?
}

\section{Allgemeine Aspekte der Medikamentenentwicklung in der Pädiatrie}

Das Fehlen von qualitativ hochwertigen und in klinischen Prüfungen in der pädiatrischen Population getesteten Arzneimitteln hat dazu geführt, dass eine Vielzahl von (nur für Erwachsene) zugelassenen Arzneimitteln im Kindesalter nicht angewendet werden können und damit Kindern potenziell wirksame Arzneimittel vorenthalten werden [1,2]. Gleichzeitig ist es jedoch ethisch schwer vertretbar, die für Kinder nicht zugelassenen Arzneimittel, deren Wirksamkeit und Unbedenklichkeit in der pädiatrischen Population durch kontrollierte klinische Prüfungen nicht eindeutig belegt sind, Kindern vorzuenthalten, wenn der Bedarf einer Arzneimitteltherapie medizinisch dringend angezeigt ist. [3]

Vor dem Hintergrund der unzureichenden Anzahl von zugelassenen Arzneimitteln, die speziell für die Anwendung in der Pädiatrie geprüft wurden, wurde in der Europäischen Union (EU) am 26. Dezember 2006 die Verordnung 1901/2006 (EG) vom Europäischen Parlament und Europäischen Rat verabschiedet (nachfolgend „Kinderarzneimittelverordnung“). Sie trat am 27. Januar 2007 in Kraft [4].

Ziel dieser Verordnung ist es, die Entwicklung von Kinderarzneimitteln zu fördern und mehr Informationen über die Verwendung dieser Arzneimittel in den Zulassungsdokumenten bereitzustellen.

Das Pädiatrische Komitee (PDCO) der Europäischen Arzneimittel-Agentur (EMA) und die EMA selbst haben bei der Umsetzung der Verordnung eine zentrale Rolle [5]. Pharmazeutische Unternehmer müssen dem PDCO für jedes neue, bisher nicht in der EU zugelassene Arzneimittel ein pädiatrisches Prüfkonzept (paediatric investigation plan, PIP) vorlegen, in dem das Konzept der Arzneimittelentwicklung für die Anwendung in der Pädiatrie zu beschreiben ist. Eine Ausnahme von der Verpflichtung der Arzneimittelentwicklung für Kinder kann vom PDCO durch Genehmigung einer Freistellung (Waiver) in der beantragten Indikation gewährt werden.

Ohne endgültige Zustimmung (Opinion) des PDCO zum entsprechenden Entwicklungskonzept (PIP oder Waiver) und Beleg über die Einhaltung der Vereinbarungen (Compliance Check) können diese Arzneimittel auch nicht für Erwachsene zugelassen werden [4].

Ferner schreibt die Kinderarzneimittelverordnung vor, dass die Ergebnisse der klinischen Studien, die basierend auf den Vorgaben der genehmigten PIPs durchgeführt wurden, veröffentlicht werden müssen. Die getroffenen Entscheidungen im Zulassungsverfahren bezüglich Indikationen, Kontraindikationen oder Warnhinweisen müssen in Absprache mit der zulassenden Behörde in der Produktinformation für diese Arzneimittel berücksichtigt werden.

Die Veröffentlichung ist unabhängig vom Ausgang der Studien und der Bewertung im Sinne der Zulassung für die pädiatrische Population. Damit soll Transparenz für alle Informationen aus der pädiatrischen Arzneimittelentwicklung für die Bevölkerung und die behandelnden Ärzten hergestellt werden.

Die Erfolge der Umsetzung der Kinderarzneimittelverordnung seit 2007 wurden in einem Bericht der Europäischen Kommission am 26.10.2017 [6] veröffentlicht und sind in Kürze in den folgenden Abschnitten dargestellt.
Der Bericht gibt einen umfassenden Überblick über die Auswirkungen der Kinderarzneimittelverordnung in Bezug auf die Entwicklung von Arzneimitteln für Kinder zehn Jahre nach ihrem Inkrafttreten. Darüber hinaus hat die EU-Kommission eine Untersuchung veranlasst, die den wirtschaftlichen Effekt der Kinderarzneimittelverordnung für die pharmazeutische Industrie und in den nationalen Gesundheitssystemen der EU-Mitgliedsländer untersucht hat.

Mit der Veröffentlichung des Berichts geht ein mehrjähriges Projekt zu Ende, das vom PDCO und den Mitarbeitern der Europäischen Arzneimittelagentur (EMA) kontinuierlich sowie durch die Zusammenstellung der Informationen für den EMA/PDCO 10-Jahresbericht unterstützt wurde [7].

\section{Auswertung 10 Jahre Kinder- arzneimittelverordnung}

Vom Juli 2007 bis Dezember 2016 wurden vom PDCO insgesamt 950 Entwicklungspläne (PIPs) mit den jeweiligen Antragstellern diskutiert und in einer finalen PIP-Opinion (PIP-Entscheidung) vereinbart. Als Ergebnis dieser PIPs wurden in der EU insgesamt 221 neue Arzneimittel speziell für die Anwendung bei Kindern einschließlich einer geeigneten Darreichungsform zugelassen.

Die nachfolgende $>$ Tab. 1 zeigt deutlich den Einfluss, den eine gesetzliche Verpflichtung zur Durchführung von Zulassungsstudien bei Kindern bewirkt hat.

- Tab. 1 Vergleich von Regionen mit und ohne gesetzliche Verpflichtung zur Durchführung von Zulassungsstudien für Kinder.

\begin{tabular}{|l|l|l|l|}
\hline $\begin{array}{l}\text { Region mit } \\
\text { Referenzzeitraum } \\
\mathbf{2 0 0 7 - 2 0 1 6}\end{array}$ & $\begin{array}{l}\text { Neue Pädiatrische } \\
\text { Zulassungen }\end{array}$ & $\begin{array}{l}\text { Neue/zusätzliche } \\
\text { Pädiatrische Indi- } \\
\text { kationen (Ände- } \\
\text { rungsanzeigen) }\end{array}$ & $\begin{array}{l}\text { Gesamtzahl der } \\
\text { pädiatrischen Zu- } \\
\text { lassung und Än- } \\
\text { derungsanzeigen }\end{array}$ \\
\hline EU & 80 & 141 & 221 \\
\hline USA & 76 & 173 & 249 \\
\hline Canada & 38 & 107 & 145 \\
\hline Japan & 12 & 38 & 50 \\
\hline
\end{tabular}




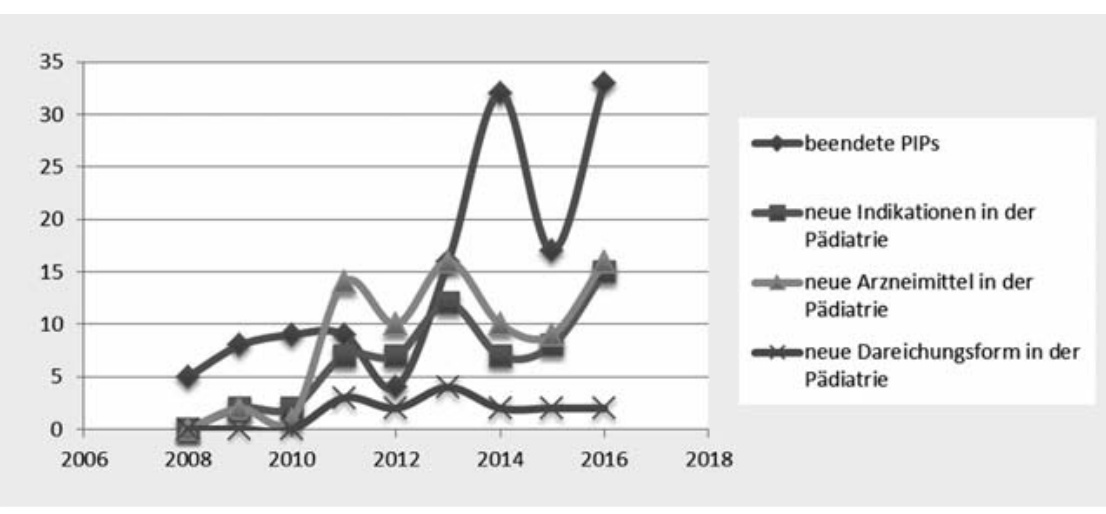

- Abb. 1 Status der Arzneimittelentwicklungen gemäß PIP Opinion.
Korrespondenzadresse

\section{Dr. med. Dirk Mentzer}

Paul Ehrlich Institut

Paul-Ehrlich-Str. 51-59

63225 Langen

dirk.mentzer@pei.de

Literatur

[1] Palmaro A, Bissuel R, Renaud N et al. Off-label prescribing in pediatric outpatients. Pediatrics 2015; 135: 49-58

[2] Magalhães ], Rodrigues AT, Roque F et al. Use of off-label and unlicenced drugs in hospitalised paediatric patients: a systematic review. Eur J Clin Pharmacol 2015; 71: 1-13

[3] Kurz R, Gill D, Mjones S; Ethics Working Group of Confederation of European Specialists in Paediatrics. Ethical issues in the daily medical care of children. Eur J Pediatr 2006; 165: 83-86

die Regionen USA und EU gesetzlich geregelte Verpflichtungen für Antragsteller einer Arzneimittelzulassung, entsprechende Zulassungen auch für Kinder zu beantragen und daher deutlich mehr pädiatrische Zulassungen.

Gemessen an dem 10-jährigen Zeitraum wirken diese Zahlen auf den ersten Blick nicht überwältigend. Berücksichtigt man aber eine durchschnittliche Entwicklungszeit von der ersten Testung im Menschen bis zur Zulassung eines Arzneimittels von ca. sieben Jahren [8], dann ist anzunehmen, dass in den nächsten Jahren die Anzahl der Arzneimittelzulassungen für die Anwendung bei Kindern weiter steigt.

\section{Fazit}

Der 10-Jahres-Bericht der EU-Kommission beschreibt die positiven Auswirkungen der Kinderarzneimittelverordnung auf die Entwicklung von Kinderarzneimitteln in der EU. Die Verordnung hat dafür gesorgt, dass die Entwicklung der Kinderarzneimittel zu einem integralen Bestandteil der Gesamtentwicklung von Arzneimitteln wurde.

Die Durchführung von klinischen Studien in der Pädiatrie zur Erforschung von Wirksamkeit und Unbedenklichkeit von Arzneimitteln ist seit Einführung der Kinderarzneimittelverordnung ein verpflichtender Bestandteil für die Zulassung neuer Arzneimittel in der EU und ist damit zu einem evidenzbasierten Bestandteil für die Be- wertung der Nutzen-Risiko-Abwägung im Zulassungsantrag geworden.

Darüber hinaus können basierend auf den Ergebnissen aus den kontrollierten klinischen Studien bestehende Wissenslücken in der Behandlung von Kindern weiter geschlossen werden und damit die Arzneimitteltherapie in der Pädiatrie sicherer gemacht werden.

Dieses Ergebnis wäre ohne eine spezifische Gesetzgebung nicht erreicht worden, was aus dem Vergleich zwischen den Regionen mit Gesetzgebung (USA und EU) und ohne Gesetzgebung (Japan und Kanada) deutlich wird.

Ferner wurde in einer separaten Untersuchung zur finanziellen Auswirkung der Kinderarzneimittelverordnung beschrieben, dass auch aus sozioökonomischer Sicht die Kinderarzneimittelverordnung insgesamt positive Ergebnisse für Zulassungsinhaber und die nationalen Gesundheitssysteme geliefert hat [9].

\section{Interessenkonflikte}

Der Autor gibt an, dass kein Interessenkonflikt besteht.

\section{Autor}

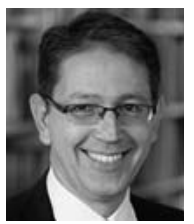

Dirk Mentzer

Paul Ehrlich Institut, Langen
[4] Regulation (EC) No 1901/2006 of the European Parliament and of the Council of 27 December 2006 on medicinal products for paediatric use (27.12.2006). Im Internet: http:// ec.europa.eu/health/files/eudralex/vol-1/ reg_2006_1901/reg_2006_1901_en.pdf; Stand: 11.09 .2018

[5] EMA Homepage mit Information zum Pädiatrischen Komitee (16.09.2010). Im Internet: http://www.ema.europa.eu/docs/ en_GB/document_library/Regulatory_ and_procedural_guideline/2009/10/ WC500004754.pdf; Stand: 11.09.2018

[6] State of Paediatric Medicines in the EU - Report from the Commission to the European Parliament and the Council (26.10.2017). Im Internet: https://ec.europa.eu/health/ sites/health/files/files/paediatrics/docs/ 2017_childrensmedicines_report_en.pdf; Stand: 11.09 .2018

[7] A technical 10-year report to the European Commission prepared by the European Medicines Agency together with its Paediatric Committe (15.08.2017). Im Internet: https://ec.europa.eu/health/sites/health/ files/files/paediatrics/docs/paediatrics_ 10_years_ema_technical_report.pdf; Stand: 11.09.2018

[8] Paul SM, Mytelka DS, Dunwiddie CT et al. How to improve R\&D productivity: the pharmaceutical industry's grand challenge. Nat Rev Drug Discov. 2010 Mar;9(3):203-14.

[9] A study on the economic impact of the Paediatric Regulation (Dec. 2016). Im Internet: https://ec.europa.eu/health/ sites/health/files/files/paediatrics/docs/ paediatrics_10_years_economic_study.pdf; Stand: 11.09 .2018 